\title{
Jeřabek Hyperbola of a Triangle in an Isotropic Plane
}

\author{
Jeřabek Hyperbola of a Triangle in an Isotropic \\ Plane

\section{ABSTRACT} \\ In this paper, we examine the Jeřabek hyperbola of an \\ allowable triangle in an isotropic plane. We investigate \\ different ways of generating this special hyperbola and de- \\ rive its equation in the case of a standard triangle in an \\ isotropic plane. We prove that some remarkable points \\ of a triangle in an isotropic plane lie on that hyperbola \\ whose centre is at the Feuerbach point of a triangle. We \\ also explore other interesting properties of this hyperbola \\ and its connection with some other significant elements of \\ a triangle in an isotropic plane.
}

Key words: allowable triangle, standard triangle, Jeřabek hyperbola

MSC2010: 51N25

In Euclidean geometry, the Jeřabek hyperbola of a triangle $A B C$ is its circumscribed rectangular hyperbola, which is the isogonal image of the Euler line of this triangle. This hyperbola is generated by the centre of homology of the triangle $A B C$ and the homothetic triangle to its tangential triangle $A_{t} B_{t} C_{t}$ with respect to the circumcentre of the triangle $A B C$. In Euclidean geometry, the Feuerbach hyperbola of a triangle $A B C$ is its circumscribed rectangular hyperbola with the centre at the Feuerbach point $\Phi$ of this triangle. We will show that an analogous hyperbola exists in an isotropic plane, which unites the aforementioned properties and some other properties of these two hyperbolas. It is a special hyperbola obtained in [10], i.e., the Jeřabek hyperbola of an allowable triangle in an isotropic plane.

In an isotropic plane, a triangle is allowable if none of its sides is an isotropic line. Each allowable triangle in an isotropic plane can be set by a suitable choice of coordinates in the so-called standard position, where its circumscribed circle has the equation $y=x^{2}$, and its vertices

\begin{abstract}
Jeřabekova hiperbola trokuta u izotropnoj ravnini
\section{SAŽETAK}

U radu proučavamo Jeřabekovu hiperbolu dopustivog trokuta u izotropnoj ravnini. Istražujemo različite načine generiranja ove specijalne hiperbole $\mathrm{i}$ izvodimo njenu jednadžbu u slučaju standardnog trokuta. Dokazujemo da neke značajne točke trokuta u izotropnoj ravnini leže na toj hiperboli čiji je centar u Feuerbachovoj točki trokuta. Proučavamo i neka druga zanimljiva svojstva ove hiperbole i njezinu vezu s nekim značajnim elementima trokuta u izotropnoj ravnini.
\end{abstract}

Ključne riječi: dopustivi trokut, standardni trokut, Jeřabekova hiperbola

are of the form $A=\left(a, a^{2}\right), B=\left(b, b^{2}\right), C=\left(c, c^{2}\right)$, while $a+b+c=0$ (see [11]). By using the abbreviations

$p=a b c, \quad q=b c+c a+a b$,

we can get some useful expressions, e.g., $a^{2}+b^{2}+c^{2}=$ $-2 q$, as well as, $q=b c-a^{2},(c-a)(a-b)=2 q-3 b c$, and identities derived therefrom by a cyclic permutation of $a, b$ and $c$.

In [6], it is proved that the Brocard angle of a standard triangle $A B C$ is given by the formula

$\omega=-\frac{1}{3 q}(b-c)(c-a)(a-b)$.

We will start with the following theorem:

Theorem 1 Let $A_{t} B_{t} C_{t}$ be a tangential triangle of an allowable triangle and $A^{\prime} B^{\prime} C^{\prime}$ the triangle obtained from the triangle $A_{t} B_{t} C_{t}$ by any translation into an isotropic direction. The triangles $A B C$ and $A^{\prime} B^{\prime} C^{\prime}$ are homologic, i.e., the lines $A A^{\prime}, B B^{\prime}$ and $C C^{\prime}$ pass through a point $T$, and the points $B C \cap B^{\prime} C^{\prime}, C A \cap C^{\prime} A^{\prime}, A B \cap A^{\prime} B^{\prime}$ lie on one line $\mathcal{T}$ (Figure 1). 
Proof. By [1], e.g. $A_{t}=\left(-\frac{a}{2}, b c\right)$, and then

$A^{\prime}=\left(-\frac{a}{2}, t+b c\right), \quad B^{\prime}=\left(-\frac{b}{2}, t+c a\right)$,

$C^{\prime}=\left(-\frac{c}{2}, t+a b\right)$,

where $t$ is a perimeter. The line with the equation

$y=-\frac{2(t+q)}{3 a} x+\frac{2 t}{3}-\frac{q}{3}+b c$

passes through the point $A=\left(a, a^{2}\right)$ and the point $A^{\prime}$ from (1) because we get

$$
\begin{aligned}
& -\frac{2(t+q)}{3 a} \cdot a+\frac{2 t}{3}-\frac{q}{3}+b c=b c-q=a^{2} \\
& -\frac{2(t+q)}{3 a} \cdot\left(-\frac{a}{2}\right)+\frac{2 t}{3}-\frac{q}{3}+b c=t+b c
\end{aligned}
$$

and it is the line $A A^{\prime}$. It passes through the point

$T=\left(\frac{3 p}{2(t+q)}, \frac{1}{3}(2 t-q)\right)$

because we get

$$
\begin{aligned}
&- \frac{2(t+q)}{3 a} \cdot \frac{3 p}{2(t+q)}+\frac{2 t}{3}-\frac{q}{3}+b c \\
&=-\frac{p}{a}+b c+\frac{1}{3}(2 t-q)=\frac{1}{3}(2 t-q),
\end{aligned}
$$

and analogously, the lines $B B^{\prime}$ and $C C^{\prime}$ pass through the point $T$. The line with the equation

$y=2 a x+t-a^{2}$

passes through the points $B^{\prime}$ and $C^{\prime}$ from (1) because e.g. for the point $B^{\prime}$ we get

$2 a\left(-\frac{b}{2}\right)+t-a^{2}=t+c a$.

Therefore this is the line $B^{\prime} C^{\prime}$. From its equation (3) and the equation $y=-a x-b c$ of the line $B C$ for the abscissa $x$ of the point $B C \cap B^{\prime} C^{\prime}$ we get the equation $3 a x=a^{2}-t-b c$ with the solution $x=-\frac{1}{3 a}(t+q)$, and then the equation of the line $B C$ implies $y=\frac{1}{3}(t+q)-b c$. So we get

$B C \cap B^{\prime} C^{\prime}=\left(-\frac{1}{3 a}(t+q), \frac{1}{3}(t+q)-b c\right)$.

This point lies on the line $\mathcal{T}$ with the equation

$\mathcal{T} \quad \ldots \quad y=\frac{3 p}{t+q} x+\frac{t+q}{3}$ because of

$\frac{3 p}{t+q} \cdot\left(-\frac{1}{3 a}\right)(t+q)+\frac{t+q}{3}=\frac{1}{3}(t+q)-b c$,

and the line $\mathcal{T}$ also passes through the analogous points $C A \cap C^{\prime} A^{\prime}$ and $A B \cap A^{\prime} B^{\prime}$.

Corollary 1 In the case of a standard triangle $A B C$, the point $T$ and the line $\mathcal{T}$ are given by formulas (2) and (4).

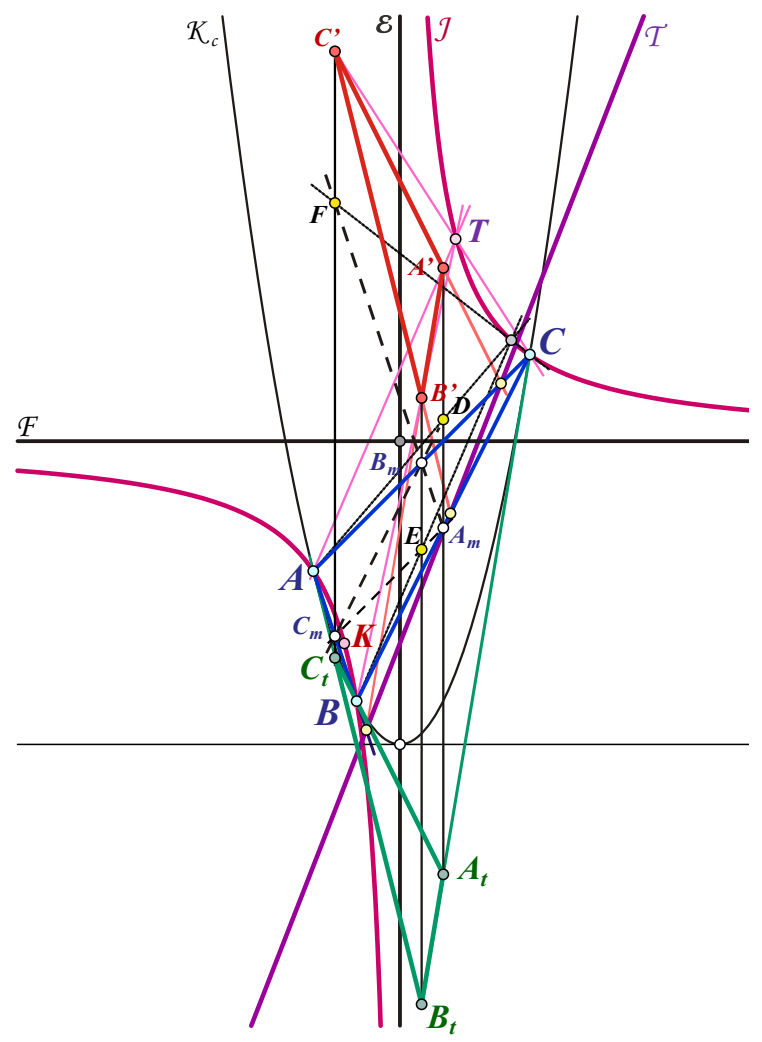

Figure 1.

Theorem 2 The point $T$ from Theorem 1 describes one special hyperbola $\mathcal{I}$ (Figure 1), which in the case of a standard triangle $A B C$ has the equation

$x y+q x-p=0$.

Proof. The point $T$ from (2) describes the curve $\mathcal{I}$ with the parametric equation

$x=\frac{3 p}{2(t+q)}, \quad y=\frac{1}{3}(2 t-q)$.

This implies firstly $y+q=\frac{2}{3}(t+q)$, and then $x(y+q)=p$, i.e. the equation (5) is written in the form $y=\frac{p}{x}-q$, it implies that the curve $\mathcal{I}$ has an isotropic asymptote with the 
equation $x=0$, and by [11], it is the Euler line of a triangle $A B C$. Its nonisotropic asymptote is given by the equation $y=-q$, and by [1], it is the Feuerbach line of that triangle. The curve $\mathcal{I}$ is a special hyperbola with the centre $(0,-q)$, and by [1], it is the Feurbach point $\Phi$ of a triangle $A B C$.

It is shown in [10] that a hyperbola with the equation (5) is the isogonal image of the Euler line of a triangle $A B C$ and it is called the Jeřabek hyperbola of that triangle.

Corollary 2 The Jerrabek hyperbola of an allowable triangle $A B C$ is the isogonal image of its Euler line and this line is its isotropic asymptote, while its nonisotropic asymptote is the Feuerbach line of a triangle $A B C$, and its centre is the Feuerbach point of that triangle (Figure 1). The Jerabek hyperbola of a standard triangle ABC has the equation (5).

Inserting $y=x^{2}$ in (5), we get the equation $x^{3}+q x-p=0$ for the abscissa of the intersection of the hyperbola $\mathcal{I}$ with the circumscribed circle of the triangle $A B C$, as the abscissas $a, b, c$ of the points $A, B, C$ satisfy this equation, we obtain:

Corollary 3 The Jeřabek hyperbola of a triangle is circumscribed to this triangle, i.e., it passes through its vertices (Figure 1).

Inserting $t=0$ in (6) we get $x=\frac{3 p}{2 q}, y=-\frac{q}{3}$ while with $t=-\frac{3}{2} q, x=-\frac{3 p}{q}, y=-\frac{4}{3} q$ is obtained. It follows that the hyperbola $\mathcal{I}$ passes through the points

$K=\left(\frac{3 p}{2 q},-\frac{q}{3}\right), \quad \Gamma=\left(-\frac{3 p}{q},-\frac{4}{3} q\right)$,

being, by [8] and [2], the symmedian centre and the Gergonne point of a triangle $A B C$. Further, with $t=-\frac{3}{2} q$ in (1) we get the point $A^{\prime}=\left(-\frac{a}{2},-\frac{3}{2} q+b c\right)$. This point lies on the midline $B_{m} C_{m}$ of a triangle $A B C$, which, by [11], has the equation $y=-a x+\frac{b c}{2}-q$ and

$-a\left(-\frac{a}{2}\right)+\frac{b c}{2}-q=\frac{1}{2}(b c-q)+\frac{b c}{2}-q=b c-\frac{3}{2} q$.

We have obtained:

Theorem 3 The Jeřabek hyperbola of a triangle passes through its symmedian centre and its Gergonne point. If the points $D, E, F$ are the intersections of the perpendicular bisectors of the sides $B C, C A$ and $A B$ of the triangle $A B C$ with its corresponding midlines, then the lines $A D, B E$ and $C F$ pass through its Gergonne point $\Gamma$.
The geometrical results of Theorems 1,2 and 3 and Corollaries 1,2 and 3 are analogous to the results in the Euclidean case (see [4] and [5]).

The following two statements are analogous to Theorems 1 and 2 .

Theorem 4 Let $\mathcal{A}^{\prime}, \mathcal{B}^{\prime}$ and $C^{\prime}$ be the lines parallel to the bisectors of the angles $A, B$ and $C$ and let the distances between them be proportional to the measure of these angles of the triangle $A B C$. These lines determine the triangle $A^{\prime \prime} B^{\prime \prime} C^{\prime \prime}$, which is homologic to the triangle $A B C$. In the case of a standard triangle $A B C$, the centre $T^{\prime \prime}$ of homology is the point

$T^{\prime \prime}=\left(-\frac{6 p t}{q(2 t-3 \omega)},-q-\frac{q}{6 t}(2 t-3 \omega)\right)$,

and the axis of homology $\mathcal{T}^{\prime \prime}$ is given by the equation

$\mathcal{T}^{\prime \prime} \ldots 3 q(3 \omega-2 t) y=9 p t x+3 q \omega t^{2}+4 q^{2} t-3 q^{2} \omega$.

Proof. By [7], the bisector of the angle $A$ has the equation $y=\frac{a}{2} x+\frac{a^{2}}{2}$, and the line $\mathcal{A}^{\prime}$ has the first of the three analogous equations

$$
\begin{aligned}
\mathcal{A}^{\prime} & \ldots & y & =\frac{a}{2} x+\frac{a^{2}}{2}+\frac{1}{2}(b-c) t, \\
\mathcal{B}^{\prime} & \ldots & y & =\frac{b}{2} x+\frac{b^{2}}{2}+\frac{1}{2}(c-a) t, \\
\mathcal{C}^{\prime} & \ldots & y & =\frac{c}{2} x+\frac{c^{2}}{2}+\frac{1}{2}(a-b) t,
\end{aligned}
$$

where $t$ is the perimeter. The point

$A^{\prime \prime}=\left(a+\frac{3 a t}{b-c}, \frac{q t}{b-c}-\frac{b c}{2}\right)$

lies on the line $\mathcal{B}^{\prime}$ and $C^{\prime}$ from (9) because e.g. for the line $\mathcal{B}^{\prime}$ we get

$$
\begin{aligned}
& \frac{b}{2}\left(a+\frac{3 a t}{b-c}\right)+\frac{b^{2}}{2}+\frac{1}{2}(c-a) t \\
& \quad=\frac{t}{2(b-c)}[3 a b+(b-c)(c-a)]+\frac{b}{2}(a+b) \\
& \quad=\frac{t}{2(b-c)} \cdot 2 q-\frac{b c}{2}=\frac{q t}{b-c}-\frac{b c}{2} .
\end{aligned}
$$

Hence, $A^{\prime \prime}=\mathcal{B}^{\prime} \cap \mathcal{C}^{\prime}$. The line with the equation

$$
y=\frac{q}{6 a t}(2 t-3 \omega) x+a^{2}-\frac{q}{6 t}(2 t-3 \omega)
$$


obviously passes through the point $A=\left(a, a^{2}\right)$, as well as through the points $A^{\prime \prime}$ and $T^{\prime \prime}$ from (10) and (7) because

$$
\begin{aligned}
& \frac{q}{6 a t}(2 t-3 \omega)\left(a+\frac{3 a t}{b-c}\right)+a^{2}-\frac{q}{6 t}(2 t-3 \omega) \\
& \quad=\frac{q}{2(b-c)}(2 t-3 \omega)+a^{2} \\
& \quad=\frac{q t}{b-c}+\frac{1}{2(b-c)} \cdot(b-c)(c-a)(a-b)+a^{2} \\
& =\frac{q t}{b-c}+\frac{1}{2}(2 q-3 b c)+b c-q=\frac{q t}{b-c}-\frac{b c}{2}, \\
& -\frac{q}{6 a t}(2 t-3 \omega) \cdot \frac{6 p t}{q(2 t-3 \omega)}+a^{2}-\frac{q}{6 t}(2 t-3 \omega) \\
& =-b c+a^{2}-\frac{q}{6 t}(2 t-3 \omega)=-q-\frac{q}{6 t}(2 t-3 \omega) .
\end{aligned}
$$

So, the point $T^{\prime \prime}$ lies on the line $A A^{\prime \prime}$, and analogously on the lines $B B^{\prime \prime}$ and $C C^{\prime \prime}$. The point

$$
L=\left(\frac{q-3 b c}{3 a}-\frac{b-c}{3 a} t, \frac{b-c}{3} t-\frac{q}{3}\right)
$$

lies on the line $B C$ with the equation $y=-a x-b c$ and on the line $\mathcal{A}^{\prime}$ from (9) because

$$
\begin{aligned}
& -a\left(\frac{q-3 b c}{3 a}-\frac{b-c}{3 a} t\right)-b c=b c-\frac{q}{3}+\frac{b-c}{3} t-b c \\
& =\frac{b-c}{3} t-\frac{q}{3} \\
& \frac{a}{2}\left(\frac{q-3 b c}{3 a}-\frac{b-c}{3 a} t\right)+\frac{a^{2}}{2}+\frac{1}{2}(b-c) t \\
& =\frac{1}{6}(q-3 b c)-\frac{1}{6}(b-c) t+\frac{1}{2}(b-c) t+\frac{1}{2}(b c-q) \\
& =\frac{1}{3}(b-c) t-\frac{q}{3}
\end{aligned}
$$

and then $L=B C \cap \mathcal{A}^{\prime}$. Accordingly, the point $L$ lies on the line $\mathcal{T}^{\prime \prime}$ with the equation (8)

$$
\begin{aligned}
& 3 q(3 \omega-2 t)\left(\frac{b-c}{3} t-\frac{q}{3}\right)-9 p t\left(\frac{q-3 b c}{3 a}-\frac{b-c}{3 a} t\right) \\
& \quad-3 q \omega t^{2}-4 q^{2} t+3 q^{2} \omega \\
&=-2(b-c) q t^{2}+3(b-c) q \omega t+2 q^{2} t-3 q^{2} \omega-3 b c(q-3 b c) t \\
& \quad+3 b c(b-c) t^{2}+(b-c)(c-a)(a-b) t^{2}-4 q^{2} t+3 q^{2} \omega \\
&=[(b-c)(3 b c-2 q)+(b-c)(c-a)(a-b)] t^{2} \\
& \quad+\left(9 b^{2} c^{2}-3 b c q-2 q^{2}\right) t-(b-c)^{2}(c-a)(a-b) t \\
&= {\left[9 b^{2} c^{2}-3 b c q-2 q^{2}+(q+3 b c)(2 q-3 b c)\right] t=0, }
\end{aligned}
$$

and the analogous points $C A \cap \mathcal{B}^{\prime \prime}$ and $A B \cap C^{\prime \prime}$ lie on the same line.
Theorem 5 The point $T^{\prime \prime}$ from Theorem 4 describes the Jeřabek hyperbola of a triangle $A B C$.

Proof. The point $T^{\prime \prime}$ from (7) describes the curve with the parametric equation

$$
x=-\frac{6 p t}{q(2 t-3 \omega)}, \quad y=-q-\frac{q}{6 t}(2 t-3 \omega),
$$

which immediately implies $x(y+q)=p$.

The geometrical results of Theorems 4 and 5 are analogous to the results in the Euclidean case (see [3]).

Theorem 6 Let $A_{i} B_{i} C_{i}$ be a contact triangle of a triangle $A B C$ and $A^{\prime \prime \prime} B^{\prime \prime \prime} C^{\prime \prime \prime}$ the triangle obtained from the triangle $A_{i} B_{i} C_{i}$ by any translation into an isotropic direction. The triangles $A B C$ and $A^{\prime \prime \prime} B^{\prime \prime \prime} C^{\prime \prime \prime}$ are homologic, and the centre $T^{\prime \prime \prime}$ of this homology describes the Jerrabek hyperbola of a triangle $A B C$.

Proof. By [1], let e.g. $A_{i}=(-2 a, b c-2 q)$; so then $A^{\prime \prime \prime}=(-2 a, t+b c-2 q)$. The line with the equation

$$
y=-\frac{t-q}{3 a} x+\frac{t}{3}-\frac{4}{3} q+b c
$$

passes through the points $A=\left(a, a^{2}\right)$ and $A^{\prime \prime \prime}$ because

$$
\begin{gathered}
-\frac{t-q}{3 a} \cdot a+\frac{t}{3}-\frac{4}{3} q+b c=b c-q=a^{2}, \\
-\frac{t-q}{3 a}(-2 a)+\frac{t}{3}-\frac{4}{3} q+b c=t+b c-2 q,
\end{gathered}
$$

and also through the point

$$
T^{\prime \prime \prime}=\left(\frac{3 p}{t-q}, \frac{1}{3}(t-4 q)\right)
$$

since

$-\frac{t-q}{3 a} \cdot \frac{3 p}{t-q}+\frac{t}{3}-\frac{4}{3} q+b c=-b c+\frac{t}{3}-\frac{4}{3} q+b c=\frac{1}{3}(t-4 q)$.

The point $T^{\prime \prime \prime}$ describes the curve with the parametric equation

$$
x=\frac{3 p}{t-q}, \quad y=\frac{1}{3}(t-q)-q,
$$

which implies $x(y+q)=p$.

The Jeřabek hyperbola is the isogonal image of the Euler line ([10]). The following property also holds.

Theorem 7 The Jerabek hyperbola of an allowable triangle is the reciprocal image of the line which is anticomplementary to its Brocard diameter, i.e., the isotropic line, which passes through the Gergonne point of that triangle. 
Proof. By [13], the reciprocity with respect to the standard triangle $A B C$ is the mapping $(x, y) \longmapsto\left(x^{\prime}, y^{\prime}\right)$, where

$x^{\prime}=-\frac{3 p q x^{2}+4 q^{2} x y-9 p y^{2}+9 p^{2} x+4 q^{3} x-12 p q y-4 p q^{2}}{q^{2} x^{2}-9 p x y-3 q y^{2}-6 p q x-4 q^{2} y+9 p^{2}}$.

It therefore maps the line with the equation $x^{\prime}=-\frac{3 p}{q}$ to the curve with the equation

$$
\begin{gathered}
q\left(3 p q x^{2}+4 q^{2} x y-9 p y^{2}+9 p^{2} x+4 q^{3} x-12 p q y-4 p q^{2}\right) \\
-3 p\left(q^{2} x^{2}-9 p x y-3 q y^{2}-6 p q x-4 q^{2} y+9 p^{2}\right)=0,
\end{gathered}
$$

and it is the equation

$$
\left(4 q^{3}+27 p^{2}\right)(x y+q x-p)=0,
$$

i.e. equation (5) since $4 q^{3}+27 p^{2} \neq 0$.

Theorem 8 If $A_{0}, B_{0}, C_{0}$ are the intersections of the corresponding sides of the orthic triangle $A_{h} B_{h} C_{h}$ and the complementary triangle $A_{m} B_{m} C_{m}$ of an allowable triangle $A B C$, then the lines $A_{h} A_{0}, B_{h} B_{0}$ and $C_{h} C_{0}$ pass through the centre $\Phi$ of the Jerabek hyperbola of a triangle $A B C$.

Proof. According to [11], the lines $B_{h} C_{h}$ and $B_{m} C_{m}$ have the equations

$$
y=2 a x+2 b c-q, \quad y=-a x+\frac{b c}{2}-q
$$

and because of $b c-q=a^{2}$, they pass through the point

$$
A_{0}=\left(-\frac{b c}{2 a}, a^{2}\right) \text {. }
$$

By [11], the point $A_{h}$ is of the form $A_{h}=(a, q-2 b c)$. The line with the equation

$$
y=-2 a x-q
$$

passes through the points $A_{h}$ and $A_{0}$ because of

$$
\begin{gathered}
-2 a^{2}-q=-2(b c-q)-q=q-2 b c, \\
-2 a\left(-\frac{b c}{2 a}\right)-q=b c-q=a^{2},
\end{gathered}
$$

which is the line $A_{h} A_{0}$. However, this line obviously passes through the point $\Phi=(0,-q)$.

An analogous statement of Theorem 8 in the Euclidean case is given in [12].

Now we will use the parametric equation (6) of the hyperbola $\mathcal{I}$.

Theorem 9 The points $T_{1}$ and $T_{2}$ of the Jeřabek hyperbola with the parametric equation (6), which correspond to the values $t_{1}$ and $t_{2}$ of the parameter $t$, have the joint line with the equation

$y=-\frac{4}{9 p}\left(t_{1}+q\right)\left(t_{2}+q\right) x+\frac{1}{3}\left(2 t_{1}+2 t_{2}+q\right)$.
Proof. The point $T$ from (2) with $t=t_{1}$ lies on the line (11) since we get

$$
\begin{gathered}
-\frac{4}{9 p}\left(t_{1}+q\right)\left(t_{2}+q\right) \cdot \frac{3 p}{2\left(t_{1}+q\right)}+\frac{1}{3}\left(2 t_{1}+2 t_{2}+q\right) \\
=-\frac{2}{3}\left(t_{2}+q\right)+\frac{1}{3}\left(2 t_{1}+2 t_{2}+q\right)=\frac{1}{3}\left(2 t_{1}-q\right) .
\end{gathered}
$$

Corollary 4 At the point $T$ given by equality (2), the Jerabek hyperbola with the equations (6) has the tangent with the equation

$$
y=-\frac{4}{9 p}(t+q)^{2} x+\frac{1}{3}(4 t+q) .
$$

Theorem 10 The point $T$ given by equality (2) is isogonal, with respect to a triangle $A B C$, to the point

$$
T^{\prime}=\left(0,-\frac{2}{3}(t+q)\right) \text {. }
$$

Proof. With $x^{\prime}=0, y^{\prime}=-\frac{2}{3}(t+q)$ we get

$$
\begin{gathered}
y^{\prime}-x^{\prime 2}=-\frac{2}{3}(t+q), \quad x^{\prime} y^{\prime}+q x^{\prime}-p=-p, \\
p x^{\prime}-q y^{\prime}-y^{\prime 2}=\frac{2}{3}(t+q) \cdot \frac{1}{3}(q-2 t),
\end{gathered}
$$

and by [10], the point isogonal to the point $T^{\prime}$ has the coordinates

$$
\begin{gathered}
x=\frac{x^{\prime} y^{\prime}+q y^{\prime}-p}{y^{\prime}-x^{\prime 2}}=\frac{3 p}{2(t+q)}, \\
y=\frac{p x^{\prime}-q y^{\prime}-y^{\prime 2}}{y^{\prime}-x^{\prime 2}}=\frac{1}{3}(2 t-q),
\end{gathered}
$$

i.e., that is the point $T$ from (2).

Theorem 11 Let $T_{1}, T_{2}$ and $T_{3}$ be the points on the Jerabek hyperbola with the equations (6), which correspond to the values $t_{1}, t_{2}$ and $t_{3}$ of the perimeter $t$, and let $T_{3}^{\prime}$ be the point isogonal to the point $T_{3}$ with respect to the triangle $A B C$. The points $T_{1}, T_{2}$ and $T_{3}^{\prime}$ are collinear if and only if

$t_{1}+t_{2}+t_{3}=-\frac{3}{2} q$

Proof. The point $T_{3}^{\prime}$ given by the equality (13) with $t=t_{3}$ lies on the line $T_{1} T_{2}$ with equation (11) supposing that

$$
-\frac{2}{3}\left(t_{3}+q\right)=\frac{1}{3}\left(2 t_{1}+2 t_{2}+q\right)
$$

that is the condition (14).

Symmetry by $t_{1}, t_{2}$ and $t_{3}$ of the condition (14) gives the following statement. 
Corollary 5 Let $T_{1}, T_{2}$ and $T_{3}$ be the points on the Jeřabek hyperbola with the equations (6), which correspond to the values $t_{1}, t_{2}$ and $t_{3}$ of the perimeter $t$ so that the equality (14) holds, and let $T_{1}^{\prime}, T_{2}^{\prime}$ and $T_{3}^{\prime}$ be the points on the Euler line of the triangle $A B C$ isogonal, with respect to that triangle, to the points $T_{1}, T_{2}$ and $T_{3}$. Then the points $T_{1}^{\prime}, T_{2}, T_{3}$; $T_{1}, T_{2}^{\prime}, T_{3} ; T_{1}, T_{2}, T_{3}^{\prime}$ are the triples of the collinear points, i.e., $T_{1}, T_{1}^{\prime} ; T_{2}, T_{2}^{\prime} ; T_{3}, T_{3}^{\prime}$ are the pairs of the opposite vertices of one complete quadrilateral.

The points $\Phi=(0,-q)$ and $G=\left(0,-\frac{2}{3} q\right)$ lie on the line (11) under the condition

$$
\frac{1}{3}\left(2 t_{1}+2 t_{2}+q\right)=-q, \quad \frac{1}{3}\left(2 t_{1}+2 t_{2}+q\right)=-\frac{2}{3} q
$$

respectively.

Hence, we get:

Corollary 6 Points $T_{1}$ and $T_{2}$ on the Jeřabek hyperbola with the equations (6), which correspond to the values $t_{1}$ and $t_{2}$ of the perimeter $t$, are diametrically opposite on this hyperbola supposing that $t_{1}+t_{2}=-2 q$, and they are collinear with the centroid $G$ of the triangle $A B C$ under the condition $t_{1}+t_{2}=-\frac{3}{2} q$.

The value of the perimeter $t$, which is associated with the point $A$ on the hyperbola (6) follows from the equality

$$
\frac{3 p}{2(t+q)}=a
$$

i.e., the equality $2(t+q)=3 b c$, and then $t=\frac{3}{2} b c-q$. With this value of $t$ the right-hand side of the equation (12) gets the form

$$
-\frac{4}{9 p}\left(\frac{3}{2} b c\right)^{2} x+\frac{1}{3}(6 b c-3 q)=-\frac{b c}{a} x+2 b c-q,
$$

and the following statement follows.

Theorem 12 Tangents $\mathcal{A}, \mathcal{B}$ and $\mathcal{C}$ of the Jerabek hyperbola of a standard triangle $A B C$ at its vertices $A, B$ and $C$ have the equations

$$
\begin{aligned}
& y=-\frac{b c}{a} x+2 b c-q, \quad y=-\frac{c a}{b} x+2 c a-q, \\
& y=-\frac{a b}{c} x+2 a b-q .
\end{aligned}
$$

Theorem 13 If $A_{i} B_{i} C_{i}$ and $A_{h} B_{h} C_{h}$ are the contact triangle and the orthic triangle of an allowable triangle $A B C$, respectively, then the points $D=B_{i} C_{i} \cap B_{h} C_{h}, E=C_{i} A_{i} \cap$ $C_{h} A_{h}$ and $F=A_{i} B_{i} \cap A_{h} B_{h}$ are the poles of the lines $B C$, $C A$, and $A B$ with respect to the Jerabek hyperbola of a triangle $A B C$.
Proof. The point

$$
D=\left(-\frac{2 b c}{a},-q-2 b c\right)
$$

lies on the lines $\mathcal{B}$ and $\mathcal{C}$ because e.g. for the line $\mathcal{B}$ with the second equation (15) we get

$$
-\frac{c a}{b}\left(-\frac{2 b c}{a}\right)+2 c a-q=2 c^{2}+2 c a-q=-2 b c-q .
$$

Therefore the point $D$ is the pole of the line $B C$. According to [11] and [1], the lines $B_{h} C_{h}$ and $B_{i} C_{i}$ have the equations

$$
y=2 a x+2 b c-q, \quad y=\frac{a}{2} x-q-b c .
$$

The point $D$ lies on these lines because of the following:

$2 a\left(-\frac{2 b c}{a}\right)+2 b c-q=-q-2 b c$,

$\frac{a}{2}\left(-\frac{2 b c}{a}\right)-b c-q=-q-2 b c$.

With $t=0$ from (12), we get the equation $y=-\frac{4 q^{2}}{9 p} x+\frac{q}{3}$ of the tangent of the Jeřabek hyperbola $\mathcal{I}$ at the symmedian centre $K$ of a triangle $A B C$. This tangent obviously passes through the point $G_{t}=\left(0, \frac{q}{3}\right)$, which is, by [1], the centroid of a tangential triangle $A_{t} B_{t} C_{t}$ of a triangle $A B C$.

With $t=-\frac{3}{2} q$ from (12), we get the equation $y=-\frac{q^{2}}{9 p} x-$ $\frac{5}{3} q$ of the tangent of a hyperbola $\mathcal{I}$ at the Gergonne point $\Gamma$ of a triangle $A B C$. This tangent obviously passes through the point $G_{t}=\left(0,-\frac{5}{3} q\right)$, which is, by [2], the centroid of the contact triangle $A_{i} B_{i} C_{i}$ of a triangle $A B C$, i.e., the Cevian triangle of a point $\Gamma$ for a triangle $A B C$.

Theorem 14 Lines parallel with the lines $A P, B P$ and $C P$ through the vertices $A_{t}, B_{t}$ and $C_{t}$ of the tangential triangle $A_{t} B_{t} C_{t}$ of a triangle $A B C$ pass through one point $P^{\prime}$ if and only if the point $P$ lies on the Jeřabek hyperbola $\mathcal{I}$ of a triangle $A B C$.

Proof. According to [1], we get e.g. $A_{t}=\left(-\frac{a}{2}, b c\right)$. Let $P=(u, v)$. The line $A P$ has the slope $\left(v-a^{2}\right):(u-a)$. Its parallel line given by the equation

$2(u-a) y=2\left(v-a^{2}\right) x+2 b c u+a v+a q-3 p$.

goes through the point $A_{t}$ because of

$$
\begin{aligned}
& \left(v-a^{2}\right)(-a)+2 b c u+a v+a q-3 p \\
& \quad=a\left(a^{2}+q\right)+2 b c u-3 p=a b c+2 b c u-3 p \\
& \quad=2(u-a) b c .
\end{aligned}
$$

The line from the equation (16) and two more analogous lines pass through one point under the condition

$$
\left|\begin{array}{lll}
u-a & v-a^{2} & 2 b c u+a v+a q-3 p \\
u-b & v-b^{2} & 2 c a u+b v+b q-3 p \\
u-c & v-c^{2} & 2 a b u+c v+c q-3 p
\end{array}\right|=0 .
$$


As e.g.

$$
\begin{aligned}
& (u-b)\left(v-c^{2}\right)-(u-c)\left(v-b^{2}\right) \\
& \quad=\left(b^{2}-c^{2}\right) u-(b-c) v-b c(b-c) \\
& \quad=-(a u+v+b c)(b-c),
\end{aligned}
$$

this condition can be written in the form

$$
\Sigma(a u+v+b c)(2 b c u+a v+a q-3 p)(b-c)=0,
$$

where $\Sigma$ represents the sum of three addends, where one is always written, and the other two are obtained therefrom by a cyclic permutation of the letters $a, b$ and $c$. The same condition can also be written as follows:

$$
\begin{aligned}
& 2 p u^{2} \Sigma(b-c)+2 u v \Sigma b c(b-c)+2 u \Sigma b^{2} c^{2}(b-c) \\
& \quad+u v \Sigma a^{2}(b-c)+v^{2} \Sigma a(b-c)+p v \Sigma(b-c) \\
& \quad+q u \Sigma a^{2}(b-c)+q v \Sigma a(b-c)+p q \Sigma(b-c) \\
& \quad-3 p u \Sigma a(b-c)-3 p v \Sigma(b-c)-3 p \Sigma b c(b-c)=0 .
\end{aligned}
$$

As we have $\Sigma(b-c)=0, \Sigma a(b-c)=0, \Sigma a^{2}(b-c)=$ $-(b-c)(c-a)(a-b), \Sigma b c(b-c)=-(b-c)(c-a)(a-$ $b)$, we obtain

$$
\begin{aligned}
& \Sigma b^{2} c^{2}(b-c)=\Sigma b c\left(a^{2}+q\right)(b-c) \\
& \quad=p \Sigma a(b-c)+q \Sigma b c(b-c)=-q(b-c)(c-a)(a-b) .
\end{aligned}
$$

Then the last condition, without the factor $(b-c)(c-$ $a)(a-b)$, has the form $-2 u v-2 q u-u v-q u+3 p=0$, i.e., in the end it has the form $u v+q u-p=0$. It means that the point $P$ lies on the hyperbola $\mathcal{I}$.

Theorem 15 With the labels from Theorem 14, the point $P^{\prime}$ describes the Jerabek hyperbola $g_{t}$ of the tangential triangle $A_{t} B_{t} C_{t}$ of a triangle $A B C$, which in the case of the standard triangle $A B C$ has the equation

$2 x y+p=0$.

Proof. Let $P^{\prime}=(u, v)$. The line $A_{t} P^{\prime}$ has the slope

$$
\frac{v-b c}{u+\frac{a}{2}}=\frac{2(v-b c)}{2 u+a} .
$$

Its parallel line given by the equation

$$
(2 u+a) y=2(v-b c) x+2 a^{2} u-2 a v-a q+3 p
$$

passes through the point $A=\left(a, a^{2}\right)$ because of

$$
\begin{aligned}
& 2(v-b c) a+2 a^{2} u-2 a v-a q+3 p=2 a^{2} u-a q+a b c \\
& \quad=2 a^{2} u+a \cdot a^{2}=(2 u+a) a^{2} .
\end{aligned}
$$

The line with the equation (18) and two more analogous lines pass through one point supposing that

$$
\left|\begin{array}{lll}
2 u+a & v-b c & 2 a^{2} u-2 a v-a q+3 p \\
2 u+b & v-c a & 2 b^{2} u-2 b v-b q+3 p \\
2 u+c & v-a b & 2 c^{2} u-2 c v-c q+3 p
\end{array}\right|=0 .
$$

As e.g.

$$
\begin{aligned}
& (2 u+b)(v-a b)-(2 u+c)(v-c a) \\
& \quad=-2 a u(b-c)+v(b-c)-a\left(b^{2}-c^{2}\right) \\
& \quad=-\left(2 a u-v-a^{2}\right)(b-c),
\end{aligned}
$$

this condition can also be written in the form

$$
\begin{aligned}
& \Sigma\left(2 a u-v-a^{2}\right)\left(2 a^{2} u-2 a v-a q+3 p\right)(b-c)=0, \\
& 4 u^{2} \Sigma a^{3}(b-c)-2 u v \Sigma a^{2}(b-c)-2 u \Sigma a^{4}(b-c) \\
& \quad-4 u v \Sigma a^{2}(b-c)+2 v^{2} \Sigma a(b-c)+2 v \Sigma a^{3}(b-c) \\
& \quad-2 q u \Sigma a^{2}(b-c)+q v \Sigma a(b-c)+q \Sigma a^{3}(b-c) \\
& \quad+6 p u \Sigma a(b-c)-3 p v \Sigma(b-c)-3 p \Sigma a^{2}(b-c)=0 .
\end{aligned}
$$

In addition to the aforementioned equations from the proof of Theorem 14, the following equations also hold:

$$
\begin{aligned}
& \Sigma a^{3}(b-c)=\Sigma a(b c-q)(b-c)=p \Sigma(b-c)-q \Sigma a(b-c)=0, \\
& \Sigma a^{4}(b-c)=\Sigma a^{2}(b c-q)(b-c) \\
& \quad=p \Sigma a(b-c)-q \Sigma a^{2}(b-c)=q(b-c)(c-a)(a-b),
\end{aligned}
$$

where, without the factor $(b-c)(c-a)(a-b)$, the last condition gets the form $2 u v-2 q u+4 u v+2 q u+3 p=0$, i.e., we have $2 u v+p=0$, meaning that the point $P^{\prime}$ lies on the curve $\mathcal{I}_{t}$ with the equation (17). This curve is a special hyperbola with the asymptotes $x=0$ and $y=0$ and its centre is at $(0,0)$, which is, by [1], the Feuerbach point $\Phi_{t}$ of a triangle $A_{t} B_{t} C_{t}$. By [1], the circumscribed circle of that triangle has the equation $y=4 x^{2}+q$. From this equation and the equation (17) we get the equation $8 x^{3}+2 q x+p=0$ for the abscissa of the intersection of these two curves. The solutions of this equation are the abscissas $-\frac{a}{2},-\frac{b}{2},-\frac{c}{2}$ of the points $A_{t}, B_{t}, C_{t}$ because of

$-\frac{a}{2}-\frac{b}{2}-\frac{c}{2}=0, \quad \frac{1}{4}(b c+c a+a b)=\frac{1}{4} q, \quad-\frac{1}{8} a b c=-\frac{1}{8} p$.

So the hyperbola $g_{t}$ is circumscribed to a triangle $A_{t} B_{t} C_{t}$, and since it has a centre $\Phi_{t}$, it is the Jeřabek hyperbola of that triangle.

The symmedian centre $K=\left(\frac{3 p}{2 q},-\frac{q}{3}\right)$ of a triangle $A B C$ lies on the hyperbola (17), which is in line with the fact that $K$ is the Gergonne point of a triangle $A_{t} B_{t} C_{t}$.

At its point $\left(x_{0}, y_{0}\right)$, hyperbola (17) has the tangent with the equation $x_{0} y+y_{0} x+p=0$, which in the case of the point 
$K=\left(\frac{3 p}{2 q},-\frac{q}{3}\right)$ gets the form

$$
\frac{3 p}{2 q} y-\frac{q}{3} x+p=0, \quad \text { i.e., } \quad y=\frac{2 q^{2}}{9 p} x-\frac{2}{3} q,
$$

and it obviously passes through the point $G=\left(0,-\frac{2}{3} q\right)$ of a triangle $A B C$. So we get:

Theorem 16 The Jeřabek hyperbola of a tangential triangle of an allowable triangle passes through its symmedian centre and at this point it touches its joint line with the centroid of the given triangle.

On the basis of Corollary 2, hyperbola $\mathcal{I}_{t}$ has the Euler line and the Feuerbach line of a triangle $A_{t} B_{t} C_{t}$ as asymptotes, i.e., the lines with the equations $x=0$ and $y=0$, by [9], the Euler and the dual Feuerbach line of a triangle $A B C$. Therefore

Corollary 7 The Jeřabek hyperbola of a tangential triangle of an allowable triangle has the Euler and the dual Feuerbach line of that triangle as asymptotes.

Theorem 17 The Jeřabek hyperbola of a tangential triangle of an allowable triangle is circumscribed to its symmetrical triangle.

Proof. According to [7], the symmetrical triangle $A_{s} B_{s} C_{s}$ of a triangle $A B C$ has e.g. the vertex $A_{s}=\left(a,-\frac{b c}{2}\right)$, which obviously satisfies equation (17).

\section{References}

[1] J. BEBAN-BRKić, R. KOLAR-ŠUPER, Z. KOLARBegović, V. Volenec, On Feuerbach's Theorem and a Pencil of Circles in the Isotropic Plane, $J$. Geom. Graph. 10 (2006), 125-132.

[2] J. Beban-Brkić, V. Volenec, Z. KolarBegović, R. KOLAR-ŠUPER, On Gergonne point of the triangle in isotropic plane, Rad Hrvat. Akad. Znan. Umjet. Mat. Znan. 515 (2013), 95-106.

[3] W. EFFEnberger, Eine systematische Zusammenfassung merkwürdiger Punkte im geradlinigen Dreieck, Zeitschr. Math. Naturwiss. Unterr. 44 (1913), 369-379.

[4] V. JeřABEK, Sur l'hyperbole $\Gamma^{\prime}$, inverse de la droite d'Euler, Mathesis 8 (1888), 81-84.

[5] V. JEŘABEK, Přispěvek $\mathrm{k}$ novějši geometrii trojúhelnika, Čas. Pěst. Math. Fys. 38 (1909), 209-2015.

[6] Z. KOLAR-Begović, R. KOLAR-ŠUPeR, V. VOLEnEC, Brocard angle of the standard triangle in an isotropic plane, Rad Hrvat. Akad. Znan. Umjet. Mat. Znan. 503 (2009), 55-60.
[7] Z. KOLAR-BEgOVIĆ, R. KOLAR-ŠUPER, $\mathrm{V}$. VOLENEC, Angle bisectors of a triangle in $I_{2}$, Math. Commun. 13 (2008), 97-105.

[8] Z. KOlAR-Begović, R. KOLAR-ŠUPER, J. BEBAN-BRKIĆ, V. VoleneC, Symmedians and the symmedian centre of the triangle in an isotropic plane, Math. Pannon. 17 (2006), 287-301.

[9] R. KOLAR-ŠUPER, Z. KOLAR-BeGOVIĆ, V. VOleneC, Dual Feuerbach theorem in an isotropic plane, Sarajevo J. Math. 18 (2010), 109-115.

[10] R. KOLAR-ŠUPER, Z. KOLAR-BEGOVIĆ, V. Volenec, J. BEBAN-BRKIĆ, Isogonality and inversion in an isotropic plane, Int. J. Pure Appl. Math. 44 (2008), 339-346.

[11] R. KOLAR-ŠUPER, Z. KOLAR-BegOVIĆ, V. Volenec, J. Beban-Brkić, Metrical Relationships in a Standard Triangle in an Isotropic Plane, Math. Commun. 10 (2005), 149-157.

[12] J.R. Musselman, Question 3029, Mathesis 51 (1937), 349.

[13] V. Volenec, Z. Kolar-Begović, R. KolarŠUPER, Reciprocity in an isotropic plane, Rad Hrvat. Akad. Znan. Umjet. Mat. Znan. 519 (2014), 171-181.

\section{Zdenka Kolar-Begović}

orcid.org/0000-0001-8710-8628

e-mail: zkolar@mathos.hr

Department of Mathematics, University of Osijek Trg Ljudevita Gaja 6, 31000 Osijek, Croatia

Faculty of Education, University of Osijek Cara Hadrijana 10, 31000 Osijek, Croatia

\section{Ružica Kolar-Šuper}

orcid.org/0000-0002-8945-2745

e-mail: rkolar@foozos.hr

Faculty of Education, University of Osijek

Cara Hadrijana 10, 31000 Osijek, Croatia

\section{Vladimir Volenec}

e-mail: volenec@math.hr

Department of Mathematics, University of Zagreb Bijenička cesta 30, 10000 Zagreb, Croatia 